ORIGINAL ARTICLE

\title{
Reflective testing: how useful is the practice of adding on tests by laboratory clinicians?
}

\author{
J R Paterson, R Paterson
}

J Clin Pathol 2004;57:273-275. doi: 10.1136/jcp.2003.010108

See end of article for authors' affiliations

......................

Correspondence to:

Dr J R Paterson, Area

Biochemistry Department,

Dumfries and Galloway

Royal Infirmary, Bankend

Road, Dumfries DG1 4AP,

UK; J.Paterson@

dgri.scot.nhs.uk

Accepted 30 June 2003

\begin{abstract}
Aims: To investigate the clinical value and practice of reflective testing, a new term to describe the practice of adding on tests when reporting or clinically authorising results.

Methods: A consultant medical biochemist collected over a calendar year (2001) copies of clinical biochemistry reports on samples to which he had added on either iron studies (iron, total iron binding capacity (TIBC), and percentage saturation), or vitamin D. Iron studies and vitamin D were added on when biochemical results, available clinical information, demographic data, and clinical experience-or combinations thereof-suggested the possibility of haemochromatosis or vitamin $D$ deficiency, respectively. The number of reports that the consultant authorised was estimated for the same calendar year. The number and percentage of raised TIBC percentage saturation and low vitamin D results from the tests that were added on were collated.

Results: Raised TIBC saturation values were found in 28 patients (18.7\% of the iron studies added on), of whom 16 were subsequently genotyped, eight having a genotype consistent with haemochromatosis. Thirty one patients with vitamin D deficiency $(23.1 \%$ of the vitamin D tests added on) were identified. Conclusions: The addition of iron studies and vitamin D tests by a laboratory clinician, when reporting, resulted in the identification of patients with haemochromatosis and vitamin D deficiency. The practice of adding on tests should be called reflective testing, because it is discretionary and is based on the clinical judgement of a laboratory clinician in the interpretation of results.
\end{abstract}

\section{METHODS}

One of us (JRP, a consultant medical biochemist) prospectively collected, over a calendar year (2001), copies of clinical biochemistry reports on samples to which he had added further investigations. He undertook four sessions (two days) of reporting each week in 2001. At that time, all paper reports were examined and signed out by the reporting biochemist. Two add on tests were specifically investigated: vitamin D (25-hydroxyvitamin D) and iron studies (iron, total iron binding capacity (TIBC), and percentage saturation). Vitamin D was measured by a radioimmunoassay method (DiaSorin, Workingham, Berkshire, UK), and iron and TIBC were determined by a colorimetric ferrozine method (Boehringer Mannheim, Mannheim, Germany). The lower limit of the serum reference interval used for winter vitamin D concentrations was $20 \mathrm{nmol} /$ litre. ${ }^{3}$ Serum vitamin D concentrations of $20 \mathrm{nmol} /$ litre or less were considered to represent vitamin D deficiency. Various TIBC percentage saturation values have been used as cutoff values when screening for haemochromatosis, from $45 \%$ to $70 \%{ }^{4}$ We selected cutoff values of $50 \%$ and $55 \%$ for women and men, respectively, above which we considered haemochromatosis to be more likely and worth investigating further.

The clinical information on the request form was available at the time of authorisation of the initial results, as were the results of previous tests. The consultant medical biochemist used the current results, previous results when available, demographic data, the clinical details provided on the request form, and his clinical experience when deciding to add on tests. The "process" of adding on the tests involved the consultant entering the additional request into the computer-writing on the report that he had added on the additional test-and then sending the report out, and taking a photocopy of the report, which was given to laboratory staff who organised the relevant analysis.

Abbreviations: NND, number of add on tests needed to obtain a diagnosis; TIBC, total iron binding capacity 
The addition of vitamin D analysis was considered when results of serum analyses showed any of the following, suggesting the possibility of vitamin D deficiency: a raised alkaline phosphatase, hypocalcaemia or adjusted calcium in the lower part of the reference interval, hypophosphataemia or a phosphate in the lower part of the reference interval. The addition of iron studies was considered when results of serum analyses suggested the possibility of haemochromatosis: raised transaminases (aspartate aminotransferase and/ or alanine aminotransferase), raised ferritin, or a combination of these. When a raised TIBC saturation value was found, the result was returned to the requesting clinician with a comment indicating the possibility of haemochromatosis and a suggestion to consider genetic testing, stating that advice could be given if required. Genetic investigation for haemochromatosis included identifying the C282Y and H63D mutations.

\section{RESULTS}

Table 1 gives an estimate of the number of reports that the medical biochemist authorised, the number and percentage of vitamin D and iron studies added on, and the number of tests added on that were found to be abnormal. The number of add on tests needed to obtain a diagnosis (NND) of vitamin D deficiency or haemochromatosis was calculated, giving NNDs of 4.3 and 18.8 for vitamin D and genetic haemochromatosis, respectively.

The identification of patients with vitamin D deficiency and haemochromatosis did lead to changes in their clinical management. Of the 26 patients with vitamin D deficiency that could be assessed more than one year later (five of the original 31 had died), 17 had been prescribed a vitamin D preparation and one patient a nutritional supplement containing vitamin D. Of the eight patients found to have a genotype consistent with haemochromatosis, five were referred to hospital for investigation and management of haemochromatosis, with four patients being venesected.

\section{DISCUSSION}

This is the first report that attempts to measure the clinical value of reflective testing - the practice of adding on further investigations when interpreting laboratory results. Specifically, we examined the reporting practice of one consultant medical biochemist with respect to adding on vitamin $\mathrm{D}$ and iron studies. He identified 31 patients with vitamin D deficiency, and 28 patients with raised TIBC saturation values, of whom 16 were subsequently genotyped, and eight had a genotype consistent with haemochromatosis (four patients homozygous for $\mathrm{C} 282 \mathrm{Y}$ and four compound heterozygotes). The use of the NND may be of value in assessing individual clinical practice involving straightforward clinical scenarios, by assessing the efficiency of a specific diagnostic strategy, much as it is used to assess the number of patients needed to be treated to obtain a given benefit in therapeutics.

\section{Take home messages}

- This is the first report that attempts to measure the clinical value of reflective testing-the practice of adding on further investigations when interpreting laboratory results

- The addition of iron studies and vitamin D tests by a laboratory clinician, when reporting, resulted in the identification of patients with haemochromatosis and vitamin D deficiency

- We suggest that the practice of adding on tests should be called reflective testing, because it is discretionary and is based on the clinical judgement of a laboratory clinician in the interpretation of results

We have compared our approach with that of reflex testing for genetic haemochromatosis, by calculating the NND for each method. In the study of reflex testing, serum samples $(\mathrm{n}=35069)$ that had raised alanine aminotransferase values $(n=1490)$ were identified and transferrin saturation added on. ${ }^{5}$ From these, 56 patients had a saturation of greater than $60 \%$ (higher cutoff value than ours), with 33 patients being genotyped. There were nine patients who were homozygous for the $\mathrm{C} 282 \mathrm{Y}$ mutation and three compound heterozygotes. This comparison of reflective and reflex testing appears to indicate that reflective testing is more efficient, with NNDs of 18.8 and 124.2, respectively. However, the concept of NND should be applied with caution. The NND for reflex testing depends crucially on the threshold value used, whereas the NND for reflective testing will depend on individual reporting practice.

\section{"We suggest that reflective testing is an important element of laboratory practice that should be further investigated and examined by others"}

The potential value of reflective testing has not previously been examined and may be important, especially with the increasing complexity and number of laboratory tests available. $^{6}$ The value of reflective testing to the requesting clinician may be important in three respects: (1) to help exclude a diagnosis, (2) to expedite a diagnosis that is fairly obvious, and perhaps more importantly, (3) to obtain a diagnosis when the original set of results is equivocal. We suggest that reflective testing is an important element of laboratory practice that should be further investigated and examined by others.

\section{ACKNOWLEDGEMENTS}

We thank Drs M J Murphy, F Toolis, and A McLelland for helpful comments on the paper, and Dr M J McMahon for coining the term, reflective testing.

Table 1 An investigation into the value of adding on iron studies and vitamin D to detect haemochromatosis and vitamin $D$ deficiency (reflective testing) in the routine clinical reporting practice of a consultant medical biochemist

\begin{tabular}{llll}
\hline Tests added on & $\begin{array}{l}\text { Total number of relevant } \\
\text { reports authorised }\end{array}$ & $\begin{array}{l}\text { Number of add on tests } \\
\text { (\% of total number of reports) }\end{array}$ & $\begin{array}{l}\text { Number of abnormal add on test results } \\
(\% \text { of the number of tests added on) }\end{array}$ \\
\hline $\begin{array}{l}\text { Iron studies } \\
\text { Vitamin D }\end{array}$ & 16798 & $150(0.89 \%)$ & $28(18.7 \%)^{*}$ \\
\hline
\end{tabular}

*Serum total iron binding capacity percentage saturation $\geqslant 55 \%$ and $50 \%$ for men $(n=21)$ and women $(n=7)$, respectively; **Serum vitamin $D$ concentration $\leqslant 20 \mathrm{nmol} /$ litre (10 patients had values $\leqslant 10 \mathrm{nmol} /$ litre). 
Authors' affiliations

J R Paterson, Area Biochemistry Department, Dumfries and Galloway Royal Infirmary, Bankend Road, Dumfries DG1 4AP, UK

R Paterson, Medical School, University of Glasgow, Glasgow, UK

\section{REFERENCES}

1 Wu AHB. Improving the utilization of clinical laboratory tests. J Eval Clin Pract 1998;4:171-81.
2 Winkel P. The application of expert systems in the clinical laboratory. Clin Chem 1989;35:1595-600.

3 Dietary reference values for food energy and nutrients for the United Kingdom, 1991. Department of Health Report on Health and Social Subjects No. 41. London: Department of Health, 1991.

4 Edwards CQ, Kushner JP. Screening for hemochromatosis. N Engl J Med 1993;328:1616-20.

5 Bhavnani M, Lloyd D, Bhattacharyya A, et al. Screening for genetic haemochromatosis in blood samples with raised alanine transferase. Gut 2000;46:707-10.

6 NHS clinical biochemistry: "a profession under siege". Bulletin of the Royal College of Pathologists 2002;120:25-8. 